\title{
Cartilage Repair by Joint Distraction and Motion Using an External Fixator for Massive Cartilage Defect
}

\author{
Tomofumi Nishino $^{1 *}$, Tomoo Ishii ${ }^{2}$, Takaji Yanai ${ }^{1}$, Fei Chang ${ }^{3}$, Naoyuki Ochiai ${ }^{1}$ \\ ${ }^{1}$ Department of Orthopaedic Surgery, University of Tsukuba, Tsukuba, Japan; ${ }^{2}$ Department of Orthopaedic Surgery, Endowed De- \\ partment of Human Resources Development for Community Medicine, Tokyo Medical University Ibaraki Medical Center, Inashiki, \\ Japan; ${ }^{3}$ Department of Orthopaedic Surgery, China-Japan Union Hospital of Jilin University, Jilin, China. \\ Email: *nishino@md.tsukuba.ac.jp
}

Received January $7^{\text {th }}, 2013$; revised February $5^{\text {th }}, 2013$; accepted February $25^{\text {th }}, 2013$

\begin{abstract}
The objective of the present study was to investigate our novel methods for the repair of massive cartilage defects by joint distraction and motion using an external fixator. In this study, we used a rabbit model of massive articular cartilage defect in order to evaluate the effectiveness of using joint distraction and motion with a ring-type external fixator. This external fixator has a hinged joint with a center of rotation along the femoral transepicondylar axis, which allows the knee joint to freely flex and extend. Mesenchymal cells from bone marrow, induced by spongialization, were differentiated into mature chondrocytes and formed hyaline-like cartilage as a result of joint distraction and movement. The transplantation of autologous cells expanded from bone-marrow-derived mesenchymal cells, concentrated autologous bone marrow aspirate, and concentrated autologous peripheral blood cells were all effective in promoting cartilage repair. The quality of the cartilage after long-term joint distraction for 6 months was inferior to that after 12 weeks. In general, weight bearing on the regenerated cartilage promoted cartilage repair, although this effect differed based on when gradual weight bearing was begun. Specifically, gradual weight bearing beginning at 9 weeks produced superior results to that beginning at 6 weeks. Our methods provide an optimal environment for cartilage regeneration.
\end{abstract}

Keywords: Cartilage Repair; Joint Distraction; Joint Motion; Spongialization; External Fixator

\section{Introduction}

Articular cartilage has a limited ability for self-repair. Pure chondral defects do not heal; rather, they usually progress to cause degeneration of the surrounding cartilage. Osteoarthritis (OA) is a major cause of impaired mobility and decreased quality of life as a result of the joint cartilage's inability to spontaneously repair itself. $\mathrm{OA}$ is associated with loss of joint motion due to a large amount of cartilage degeneration. There are a few procedures that can be used to effectively treat OA [1,2]. In particular, joint distraction using an external fixator bridging the joint is an approach that provides an alternative joint to prevent progression to OA. Several clinical studies have demonstrated that mechanical stimulation through joint distraction and/or joint motion promotes cartilage repair [3-5]. In 1978, Judet and Judet [2] reported satisfactory treatment of OA using a hinged distraction device that permits joint motion after surgical arthrolysis or arthroplasty. However, after this breakthrough report, the procedure was not further investi-

${ }^{*}$ Corresponding author. gated, and little animal experimental data are available. As such, it is unclear whether this approach promotes cartilage regeneration. Twenty years later, an interest in joint distraction was rekindled by van Valburg et al. [3]. They used joint distraction with an Ilizarov apparatus to treatment patients with OA of the ankle. Distraction for 3 months improved pain, function, and mobility in more than two-thirds of the patients over a 2-year period. Valburg et al. suggested that the creation of space and the circulation of synovial fluid allowed for increased nutriation of the cartilage through intermittent hydrostatic pressure. Furthermore, in vitro studies have shown that such fluid pressures are beneficial to cultures of human osteoarthritic cartilage [4]. The use of an external fixator for joint regeneration can control the mechanical environment. An external fixator can control weight bearing and maintain the joint structure by joint distraction. It can also act as a chamber that allows cartilage regeneration promoted by joint motion in vivo. The objective of the present study was to investigate our novel methods for the repair of massive cartilage defects by joint distraction and motion using an external fixator. 


\section{Materials and Methods}

\subsection{Experimental Animals and Operative Technique}

Sixteen-week-old male Japanese white rabbits (Tokyo Experimental Animals, Tokyo, Japan; mean weight 3.1 $\mathrm{kg}$ ) were used in our experiments. Following a 2-week acclimatization period, the rabbits were anesthetized with an intravenous injection of pentobarbital (Somnopentyl, 50 mg/kg body weight, Kyoritsu Seiyaku, Tokyo, Japan), and an Ilizarov-type half-ring external fixator with a hinge was fixed onto the left knee of each rabbit (Figure 1). The external fixator was designed to adapt to the size and anatomy of the rabbits, and it has been demonstrated to be useful in a rabbit model of cartilage defect [6]. After attaching the external fixator, the patella was everted. The cruciate and collateral ligaments were divided, and the menisci were resected. A full-thickness osteochondral defect was then made on the entire surface of the tibial plateau using an oscillating saw. A space was formed in the femorotibial joint by the resected osteochondral defect, resulting in joint distraction. After the operation, movement was unrestricted, and the rabbits were allowed free access to food and water. All rabbits were sacrificed by a lethal injection of pentobarbital sodium. This study was approved by the University Committee for Animal Experimentation.

\subsection{Evaluation}

\subsubsection{Visual Inspection}

After sacrifice, the operated left knee joint was exposed, photographed with a digital camera, and visually inspected. The proximal tibia was then resected and fixed by immersion in $10 \%$ formalin.

\subsubsection{Histological Evaluation}

We performed a histological examination of sections

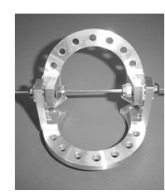

(a)

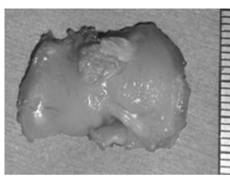

(d)

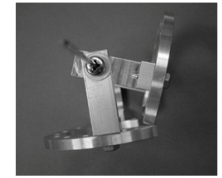

(b)

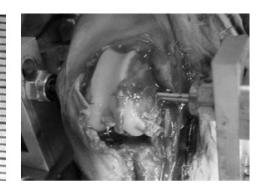

(e)

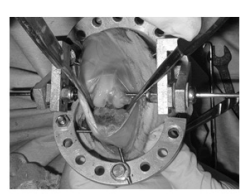

(c)

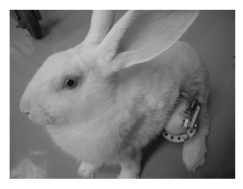

(f)
Figure 1. The rabbit experimental model. (a), (b) Ilizarovtype 2/3 ring fixator. (c), (d), (e) Total tibial plateau resection after setting the fixator using an oscillating saw $3-\mathrm{mm}$ space $=$ Joint distraction. (f) A rabbit allowed free movement in a cage. stained with Safranin-O fast green [7] and immunostained for type I collagen and type II collagen. After decalcification in EDTA, the centers of the medial and lateral tibial plateau were cut into sagittal sections, embedded in paraffin, and cut into 5 - $\mu \mathrm{m}$-thick sections. To stain proteoglycans of the cartilage matrix, the sections were stained with Safranin-O. After deparaffinization, the paraffin-embedded sections were stained with Weigert iron hematoxylin for $5 \mathrm{~min}$, counterstained with fast green for $3 \mathrm{~min}$, washed with 1\% acetic acid, and immersed in $0.1 \%$ Safranin-O. The sections were dehydrated, cleared, mounted, and examined by light microscopy. To distinguish between hyaline cartilage and fibrocartilage, the sections were immunostained for type I collagen and type II collagen as previously described by Yanai et al. [6].

\subsubsection{Histological Scoring}

Histologic evaluation was performed using the modified International Cartilage Repair Society (ICRS) scoring system [6]. This scoring system originally included 6 criteria [8], but tissue staining with type I and type II collagen antibodies was added for a total of 8 criteria. In our experiments, using mid-sagittal sections of the medial and lateral tibial plateaus, the central third of the area facing the femoral condyle was examined.

\subsubsection{Evaluation of Regenerated Tissue Area}

In our full-thickness cartilage defect model, bone, soft tissue, including fibrous tissue, fibrocartilage, and hyaline cartilage were regenerated. To quantitatively evaluate the regenerated tissue area, the sections were examined by light microscopy (BX51, Olympus), digitally imaged with a CCD camera system (DP50, Olympus) and Studio Lite (version 1.0), and analyzed with NIH Image software on a personal computer to measure tissue area. The regenerated soft tissue area, Safranin-O-positive area, and type II collagen antibody-positive area were evaluated using NIH Image. The ratio of the positive staining area to the regenerated soft tissue area was calculated for each stain. The regenerated soft tissue area did not include fibrous tissue, synovium, or regenerated meniscus attached to the joint capsule. The procedure was previously described by Yanai et al. [6].

\section{Results}

\subsection{The Process of Cartilage Repair in Our Model}

In 2005, we first reported a model of large articular cartilage defects in rabbits fitted with a hinged external fixator [6]. We evaluated the short-term effects for up to 12 weeks after the operation during which the external fixator maintained joint distraction and motion to allow 
cartilage repair. In this study, the group treated with distraction and joint immobilization had a worse outcome than the group treated with joint distraction and motion. Spongialization is advantageous due to its safety and convenience, because it does not require exogenous factors, carriers, or transplanted cells. Surprisingly, the results of this first experiment demonstrated that maintaining a suitable mechanical environment using the external fixator could promote the generation of hyaline-like cartilage (Figure 2). Indeed, hyaline-like cartilage was observed after 3 weeks and gradually matured up to 12 weeks. Starting at 6 weeks, the cells had a columnar arrangement. We proposed that the space made by distraction creates a suitable environment for culturing mesenchymal cells. Furthermore, joint motion establishes a physiologic environment whereby mechanical stress, hypoxemic stress, and nutrient diffusion promote cell growth and differentiation into articular cartilage.

\subsection{Effect of Mesenchymal Cell Transplantation}

We also evaluated the effect of joint distraction and the transplantation of autologous culture-expanded bone marrow-derived mesenchymal cells at 12 weeks in the same experiment. In the group receiving autologous culture-expanded bone marrow-derived mesenchymal cell transplantation, the morphology of the matrix, cell distribution, and the area of regenerative cartilage, as indicated by staining with Safranin-O, were superior to that of the vehicle-treated group as scaffold control group with atelocollagen gel only. However, there was no significant difference when compared to the control group, which was treated with joint distraction and motion with

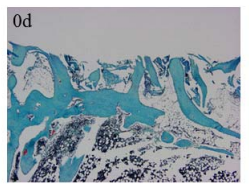

(a)

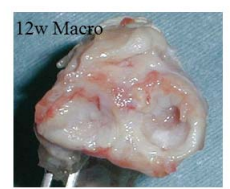

(d)

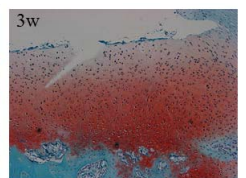

(b)

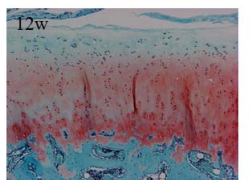

(e)

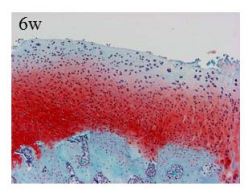

(c)

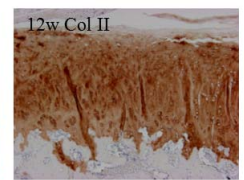

(f)
Figure 2. The process of cartilage repair in our model. (a) Bone marrow open to the articular space (mesenchymal cell invasion) immediately following the operation. (b) Differentiation into chondrocytes 3 weeks after the operation. (c) Chondrocyte proliferation and arrangement at 6 weeks. Macroscopic inspection. (d) White cartilage and meniscallike tissue at 12 weeks. (e) Hyaline-like cartilage stained with an anti-type II collagen antibody (f) at 12 weeks (Safranin-O/Fast Green, a: $\times 40$; b, c, and e: $\times 100$; Type II collagen antibody, $\mathrm{f}: \times 100)$. an external fixator. There were better results in the group treated with autologous culture-expanded bone marrow-derived mesenchymal cell transplantation compared with the vehicle-treated group. These results indicated that the transplantation of autologous culture-expanded bone marrow-derived mesenchymal cells was effective. Later, Chang, et al. [9] transplanted uncultured autologous bone marrow-derived mononuclear cells with a fibrin gel and uncultured autologous peripheral bloodderived mononuclear cells with a fibrin gel in the same model. Histological results indicated that the group transplanted with uncultured autologous bone marrowderived mononuclear cells had superior cartilage repair than the other groups, including the vehicle-treated group. The transplantation of uncultured autologous bone marrow-derived mononuclear cells contributes to articular cartilage repair; therefore, this easy and safe method is potentially viable for clinical application. However, there was no evidence that these transplanted cells remained in the joint cavity or adhered to the defect or that the newly formed cartilage was host derived.

\subsection{The Long-Term Process of Cartilage Repair in Our Model}

The long-term results are more relevant to the clinical application of this procedure. Therefore, we investigated the long-term results 6 months and 1 year after the operation [10]. The rabbits were divided into 2 groups. In the first group, joint distraction and motion with the external fixator were maintained for 6 months, after which the animals were sacrificed. In the second group, joint distraction and motion with the external fixator were maintained for 6 months, after which the external fixators were removed under sedation. The rabbits were allowed to ambulate without the external fixator for an additional 6 months and were sacrificed 1 year after surgery. Repaired cartilage remained in all rabbits. Type II collagen-positive and repaired soft tissue areas were larger in the group sacrificed 1 year after surgery than in the group in which external fixator was maintained for 6 months. In comparison with the results obtained 12 weeks after surgery [6], both areas were larger at 12 weeks after surgery than in either of the long-term groups. These findings demonstrate that the long-term persistence of repaired cartilage with this technique worsened the condition of cartilage repair, although weight bearing had a positive effect on cartilage quality.

\subsection{Effect of Weight Bearing on Cartilage Repair}

We considered that the mechanical strength of the regenerated cartilage was weak. For clinical applications in regenerative medicine, particularly in weight-bearing 
cartilage, the timing, duration, and amount of weight bearing are important factors to consider. To date, however, no studies have investigated these factors in an in vivo model. Therefore, we generated an animal model of controlled weight bearing with joint distraction and motion to investigate the relationship between weight bearing and cartilage regeneration. We developed a ring-type external fixator by modifying the previous device as well a device that allowed for controllable weight bearing and motion in rabbits [11]. This device maintains constant tension on the joint, which permits continuous passive motion while constant compression is applied to the joint space. Postoperatively, joint distraction using the external fixator and free mobility promoted cartilage regeneration at 6 and 9 weeks. To apply joint compression, the wire tension was gradually increased weekly from $0.5 \mathrm{~kg}$ to $1 \mathrm{~kg}$, and then to $2 \mathrm{~kg}$. In this manner, continuous passive motion and joint compression could be performed 2 hours daily for 3 weeks (Figure 3).

The initiation of gradual weight bearing and continuous passive motion for 6 weeks postoperatively resulted in no significant change in the histological assessment scale. The percentage of the type-II collagen-positive area was significantly larger in the gradual weight-bearing group than in the continuous passive motion group. This suggests that optimal mechanical stress, such as gradual weight bearing, may affect cartilage regeneration in vivo. When gradual weight bearing and continuous passive motion were begun 9 weeks postoperatively, the ratio of the Safranin-O staining area to the regenerated soft tissue area was significantly larger than in the other groups (Figure 4). These results demonstrate that mechanical stress, such as gradual weight bearing, is essential for the maintenance of the regenerated cartilage and

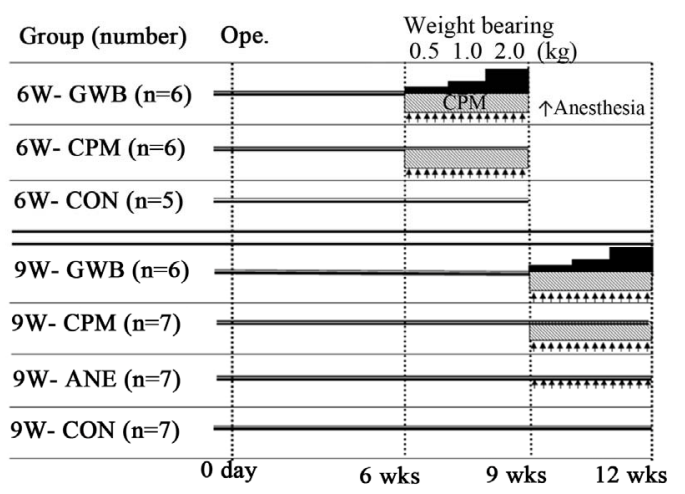

Figure 3. Start of weight bearing after 6 and 9 weeks. Gradual weight bearing (GWB) is defined as stepped-up compression to the joint every week for 3 weeks. Continuous passive motion (CPM) and GWB were examined for 2 hours every day under anesthesia (arrow) using a controller of fixator for real-time weight bearing and CPM system. CPM (continuous passive motion), ANE (anesthesia), CON (control).

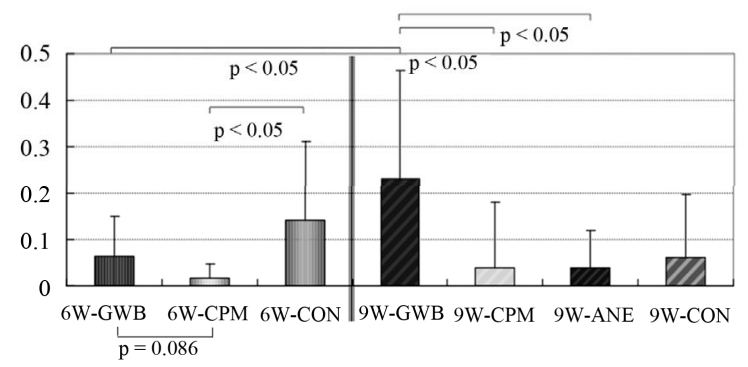

Figure 4. Ratio of Safranin-O stained area to area of regenerated soft tissue. The graph shows that gradual weight bearing (GWB) 9 weeks after joint distraction and motion using the external fixator is superior to other groups.

for its differentiation into mature cartilage. Thus, we conclude that to ensure cartilage regeneration in this model, gradual weight bearing and continuous passive motion is best begun 9 weeks after joint distraction and motion as compared to 6 weeks.

\section{Discussion}

In the present study, we demonstrate a novel animal model with an external fixator that can apply controlled pressure to the joint. This model allows for quantitative application of compression to the regenerating tissue in vivo. The use of the external fixator for joint regeneration allows for the control of the mechanical environment in the joint system. Joint distraction as tool for weightbearing control could act as chamber for cartilage regeneration in vivo. The use of a joint fixator as a tool for the control of joint movement could maintain the joint structure physiologically. This provides an optimal environment for cartilage regeneration. Its advantages include safety and convenience without the need for exogenous factors, carriers, or transplanted cells. We used a spongialization model, where cancellous bone was exposed by cutting the tibial plateau in order to differentiate cells, such as bone marrow-derived mesenchymal cells, into hyaline-like cartilage. The present findings demonstrate that the induction of mesenchymal cells to chondrocytes occurs in vivo as well as in vitro [12]. This procedure is similar in principle to the microfracture and drilling procedures for osteochondral defects. This model can be applied to OA if the mechanical environment surrounding the joint is controlled by an external fixator.

Cell transplantation is also important in cartilage regeneration. In the present study, autologous culture-expanded bone marrow-derived mesenchymal cell transplantation was effective in regenerating cartilage. Transplanted mesenchymal cells may differentiate into chondrocytes and facilitate cartilage repair. Nevertheless, for the effective clinical application of engineered tissues, the surgical handling and delivery of cell constructs must be convenient, efficient, and reliable under standard op- 
erating room conditions. Uncultured autologous bone marrow-derived mononuclear cells and uncultured autologous peripheral blood-derived mononuclear cells were also effective. The cells themselves and cytokines may be factors that improve cartilage repair by cell transplantation. However, there were no significant differences between the groups treated with joint distraction and motion using an external fixator with and without cell transplantation. From the examination of transplanted cells, we hypothesize that simply controlling the mechanical environment can maintain the cell culture space and allow joint motion with an external fixator, which could be one of the most important factors for cartilage repair and maturation.

We performed an experiment controlling weight bearing in order to identify the mechanical conditions that provide the best results for regenerated tissue. Naturally, there were different responses to mechanical stress. In this experiment, we began weight-bearing application at 6 and 9 weeks after distraction with an external fixator. Since we proposed that that response to a compressive load would be indicated by the appearance of a columnar structure, we started compression at 6 and 9 weeks. The compressive load was gradually increased at weekly intervals from $0.5 \mathrm{~kg}$ to $1 \mathrm{~kg}$, and then to $2 \mathrm{~kg}$. Given that rabbits weigh approximately 3 to $4 \mathrm{~kg}$, a $2-\mathrm{kg}$ load on one leg was considered equivalent to equal weight bearing on both legs. Gradual weight bearing and continuous passive motion 9 weeks after joint distraction and motion using the external fixator was essential for the maintenance of the regenerated cartilage and for its differentiation into mature cartilage. Therefore, as in clinical rehabilitation, we gradually increased compression. For surgeries aimed at cartilage regeneration (e.g., microfracture and autologous chondrocyte transplantation), the standard practice is to employ a postoperative rehabilitation program that includes a gradual increase in weight bearing and reaches full weight bearing by 6 to 8 weeks $[13,14]$. On the basis of the results of the present study, this approach is reasonable. We hope that our results clarify the in vivo effects. However, we have yet to clarify the timing, duration, and amount of weight bearing that is actually appropriate for regenerating cartilage. More complex studies will be required to satisfactorily resolve these issues. And we have not applied this procedure to human, yet. We consider that there are a lot of differences between rabbit and human such as cell responsibility, viability and tolerance.

Bone marrow-derived mesenchymal cells induced by spongialization differentiated into mature chondrocytes and formed hyaline-like cartilage after joint distraction and movement with a hinged external fixator. Cell transplantation of mesenchymal cells also promoted cartilage repair. The quality of the cartilage formed after long-term joint distraction for 6 months was inferior to that after 12 weeks. Weight bearing on regenerated cartilage generally promoted cartilage repair and cell maturation. Although the effect differed based on the time when gradual weight bearing was begun, our experiments showed cartilage repair at 9 weeks was superior to that at 6 weeks.

\section{Acknowledgements}

The authors did not receive and will not receive any benefits or funding from any commercial party directly or indirectly related to the subject of this article.

\section{REFERENCES}

[1] J. A. Buckwalter and H. J. Mankin, “Articular Cartilage: Tissue Design and Chondrocyte-Matrix Interactions,” Instructional Course Lectures, Vol. 47, 1998, pp. 477- 486.

[2] R. Judet and T. Judet, "The Use of a Hinge Distraction Apparatus after Arthrolysis and Arthroplasty (Author's Transl.)," Revue de Chirurgie Orthopédique et Réparatrice de L'appareil Moteur, Vol. 64, No. 5, 1978, pp. 353-365.

[3] A. A. van Valburg, P. M. van Roermund, A. C. Marijnissen, J. van Melkebeek, J. Lammens, A. J. Verbout, et al., "Joint Distraction in Treatment of Osteoarthritis: A Two-Year Follow-Up of the Ankle,” Osteoarthritis Cartilage, Vol. 7, No. 5, 1999, pp. 474-479. doi:10.1053/joca.1998.0242

[4] A. A. van Valburg, H. L. van Roy, F. P. Lafeber and J. W. Bijlsma, "Beneficial Effects of Intermittent Fluid Pressure of Low Physiological Magnitude on Cartilage and Inflammation in Osteoarthritis. An in Vitro Study," The Journal of Rheumatology, Vol. 25, No. 3, 1998, pp. 515520.

[5] R. B. Salter, D. F. Simmonds, B. W. Malcolm, E. J. Rumble, D. Mac Michael and N. D. Clements, "The Biological Effect of Continuous Passive Motion on the Healing of Full-Thickness Defects in Articular Cartilage. An Experimental Investigation in the Rabbit," Journal of Bone and Joint Surgery, Vol. 62, No. 8, 1980, pp. 1232-1251. doi:10.2106/JBJS.K.01406

[6] T. Yanai, T. Ishii, F. Chang and N. Ochiai, "Repair of Large Full-Thickness Articular Cartilage Defects in the Rabbit: The Effects of Joint Distraction and Autologous Bone-Marrow-Derived Mesenchymal Cell Transplantation,” Journal of Bone and Joint Surgery, Vol. 87, No. 5, 2005, pp. 721-729. doi:10.1302/0301-620X.87B5.15542

[7] L. Rosenberg, "Chemical Basis for the Histological Use of Safranin O in the Study of Articular Cartilage,” Journal of Bone and Joint Surgery, Vol. 53, Suppl. 2, 1971, pp. 69-82. doi:10.2106/JBJS.K.01406

[8] P. Mainil-Varlet, T. Aigner, M. Brittberg, P. Bullough, A. Hollander, E. Hunziker, et al., "Histological Assessment of Cartilage Repair: A Report by the Histology Endpoint Committee of the International Cartilage Repair Society (ICRS)," Journal of Bone and Joint Surgery, Vol. 85, Suppl. 2, 2003, pp. 45-57.

[9] F. Chang, T. Ishii, T. Yanai, H. Mishima, H. Akaogi, T. 
Ogawa, et al., "Repair of Large Full-Thickness Articular Cartilage Defects by Transplantation of Autologous Uncultured Bone-Marrow-Derived Mononuclear Cells," Journal of Orthopaedic Research, Vol. 26, No. 1, 2008, pp. 18-26. doi:10.1002/jor.20470

[10] T. Nishino, F. Chang, T. Ishii, T. Yanai, H. Mishima and N. Ochiai, "Joint Distraction and Movement for Repair of Articular Cartilage in a Rabbit Model with Subsequent Weight-Bearing," Journal of Bone and Joint Surgery, Vol. 92, No. 7, 2010, pp. 1033-1040. doi:10.1302/0301-620X.92B7.23200

[11] T. Nishino, T. Ishii, F. Chang, T. Yanai, A. Watanabe, T. Ogawa, et al., "Effect of Gradual Weight-Bearing on Regenerated Articular Cartilage after Joint Distraction and Motion in a Rabbit Model," Journal of Orthopaedic Research, Vol. 28, No. 5, 2010, pp. 600-606. doi:10.1002/jor.21016
[12] C. Y. Huang, K. L. Hagar, L. E. Frost, Y. Sun and H. S. Cheung, "Effects of Cyclic Compressive Loading on Chondrogenesis of Rabbit Bone-Marrow Derived Mesenchymal Stem Cells,” Stem Cells, Vol. 22, No. 3, 2004, pp. 313-323. doi:10.1634/stemcells.22-3-313

[13] J. R. Steadman, W. G. Rodkey and J. J. Rodrigo, “Microfracture: Surgical Technique and Rehabilitation to Treat Chondral Defects," Clinical Orthopaedics and Related Research, Vol. 391, Suppl. 391, 2001, pp. S362S369. doi:10.1097/00003086-200110001-00033

[14] M. M. Reinold, K. E. Wilk, L. C. Macrina, J. R. Dugas, E. L. Cain, "Current Concepts in the Rehabilitation Following Articular Cartilage Repair Procedures in the Knee," Journal of Orthopaedic \& Sports Physical Therapy, Vol. 36, No. 10, 2006, pp. 774-794. doi:10.2519/jospt.2006.2228 